\title{
Prediction English word concreteness ratios by their multidimensional Perceptual and Action strength norms
}

\author{
Mohsen Dolatabadi \\ Tarbiat Modares University \\ Cognitive science
}

\begin{abstract}
Many dataset resulted by participant rating for word norms also concreteness ratios are available. However infrequent word and none words concreteness rank dataset is rare. Here we used Lancaster sensorymotor word norms, to predict word concreteness ratios of Brysbaer dataset. After removing missing values and possible collinear variables, we employed a SWMLR for choosing optimum number of norms to make a prediction MLR model. Finally we validate our model using 10-fold cross-validation. The final model could predict concreteness by RMSE 0.723 and Rsquare 0.515 .
\end{abstract}

Keywords: Concreteness, prediction, perception, multiple linear regression

\section{Introduction}

Why people categorize some words concrete and the others abstract? How we can predict the concept concreteness value by their embodied properties? Embodied cognition is claim of many studies (e.g., Barsalou, 1999; Connell, 2019; Connell \& Lynott,2014b; Smith \& Gasser, 2005; Vigliocco, Meteyard,Andrews, \& Kousta, 2009; Wilson, 2002) .People 
experience world by combination of sensory-motor and action-effector perceptions, alongside this is a proven statement for mental representation of lexicons(Yao et. al,2020). As a matther of fact, a lot of studies have shown that we understand language in relation to space as if we are interacting with the world. A prime example of this type of processing is for idiomatic metaphor involving concurrently activation sensorimotor and abstract concepts brain regions(Hellmann et. al., 2013). Althought Concreteness rank of lexicons is a controversial topic in many languages, concepts which usually experinced by sensory-motor brain networks in dorsal pathway consider to be more concrete .often in linguistics studies the higher imaginibility and contextual availability determined the higher ranking for word concreteness (e.g. Montefinese,2019). Furthermore, based upon recent studies, the difference between concrete and abstract word representation in our brain allocated to inferior frontal gyros since abstract concept are more engaged in verbal regions[Wang et al. 2010]. In spite of many database for knowing frequent word concreteness ratios that have gatherd participant ratings (like Brysbaert dataset), the concreteness prediction of infrequent words also nonewords is essential for current and steel in progress demands(Chuang1 et. al.,2020) . The pseudo words composed of sound strings, have a long history of usage in psycholinguistic research. Against common belief, recent LDL(liner discriminative learning) computational model of lexical processing ponder them as semantic entities which emphasize need for expanding knowledge about their properties in future(Yu-Ying Chuang. al., 2020, ) . Moreover, many studies demonstrate dominance of special word properties in particular mental disorders like Shizophernia, Alzeimer, Parkinson and aphasia then having deep insight in pseuodoword properties would help to better disease differential diagnosis (Hoffmann,2011)

In the present work, we made stepwise multiple linear regression models to opt in the best concreteness predictors in the final models. At last we performed 10 -folds cross validation, a statistical analysis to know prediction performance of the best model.

\section{Methods}

The data used for this study involved concreteness mean likert scale values reported by Brysbares et. al(2013) and also Lancaster multimodal norms consisted of perceptual and action strengths for 40000 English words(Lynott,2019).

Since colinearity is demolishing factor for our predictive model we followed statistical criteria(eg. Dohoo et al., 1997) to detect correlated independent variables, namely, VIF (variance inflation factor) near 10 and correlation coefficient above or equal 0.9.

after removing missing values, our data dimensions were $39702 * 12$ which tested for the colinearity (coefficient $\geq 0.9$ ) among variables including dependent concreteness variable and also 11 word norms.

Multiple linear regression analysis 
MLR is one of the most used methods for determination winning combined variables for predicting outcomes.

it is linear model extension which uses only one predictor.

Concreteness $=a_{0}+a_{i} d_{i}+\cdot \ldots \cdot+a_{n} d_{n}$

which $\mathrm{a} 0$ as intercept and regeression coeffeicient are assigned by least-square methods. We used $\mathrm{R}$ software for making MLR models.

Stepwise multiple regression

The forward and backward or generally, stepwise multiple regressions used for inclusion of best predictors. This techniques begins from null model and full model to add or remove step wisely variables which causes discovery of the best fitted model by AIC values.

Cross-validation technique

The consistency and reliability of a method can be explored using the cross-validation techniques. Therefore, researchers uses two type of cross-validation including either exhaustive cross-validation involving LPO (leave percent out) and specific case of it namely LOO(leave one out) or they use non-exhaustive cross-validation involving $K$-folds cross-validation holdout method and repeated random sub-sampling validation.

In the present work we split the dataset to $20 \%$ test and $80 \%$ train data. Then we use train subset to refit our model afterward we employed test data to know our model predictive power.

Results

Modality- specific norms are useful for performance prediction of participants in lexical decision task (Lynott et. al. ,2019) in addition to anticipating word properties , specially concreteness and imaginability scale.

Here we did further analyses to know which modality-specific variables of Lancaster dataset are more meaningful to concreteness prediction which is an well-known challenge to discriminate concreteness and abstractness (for review refer to Montefinese,2019) and its application in detection properties of unpredicted and new pseudo words is an ongoing field(Chuang1 et. al. ,2020) 
Since collinearity among variables degrades model performance, at first we computed correlation coefficients for all of variables that resulted in no correlated variables.

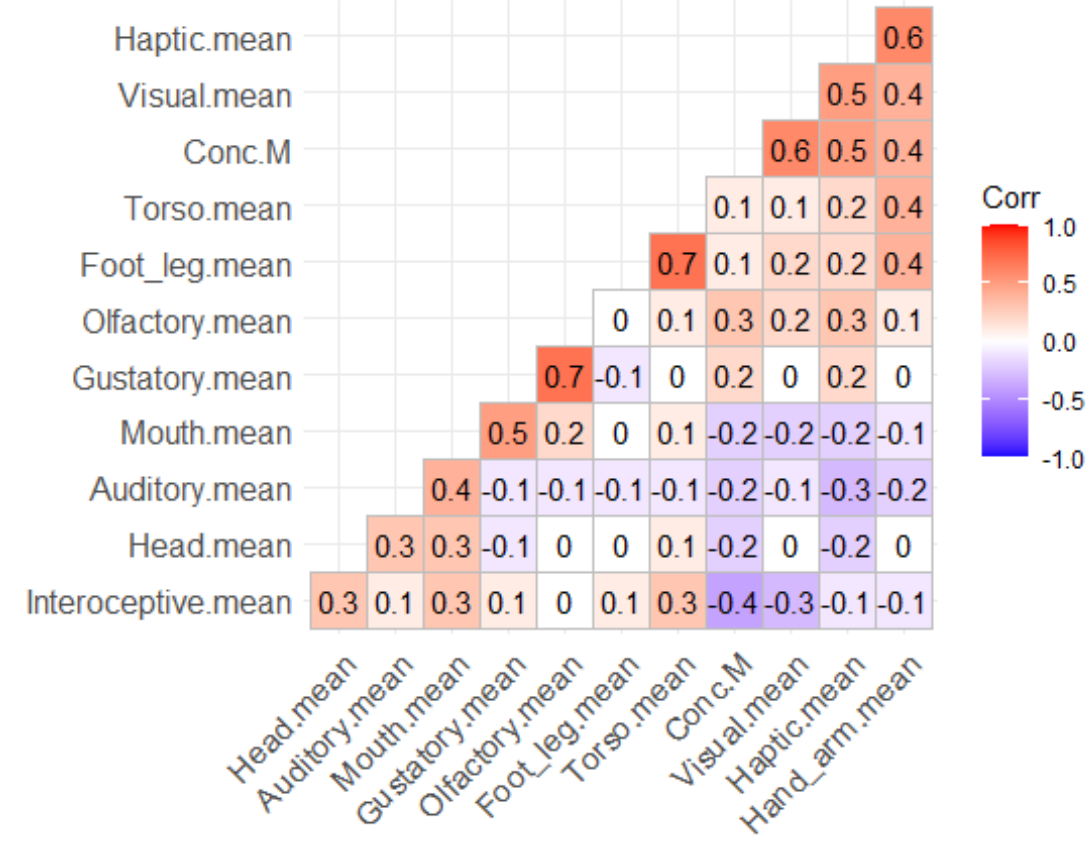

Fig.1.correlation matrix for all variables

Residual standard error of calculated stepwise model was 0.7235 on 39691 degrees of freedom for the equation (1) . Alongside, for the model multiple R-squared was 0.5153 also adjusted R-squared was equal to 0.5152 revealing that our calculated model was moderately in good.

$\mathrm{Eq}(1)$ : 
concreteness $=1.890-0.025$ Auditory.mean +0.025 Gustatory.mean +0.294 Haptic. mean + 0.214 Olfactory.mean- 0.359 Interoceptive.mean +0.337 Visual.mean -0.053 Footleg.mean+0.074Hand-arm.mean -0.028 Head.mean +0.210 Torso.mean,

After calculating VIF for our model (Fig.2) again, none of variables had near 10 quantity , what was guiding us to approve the all independent variable in step-wised model.

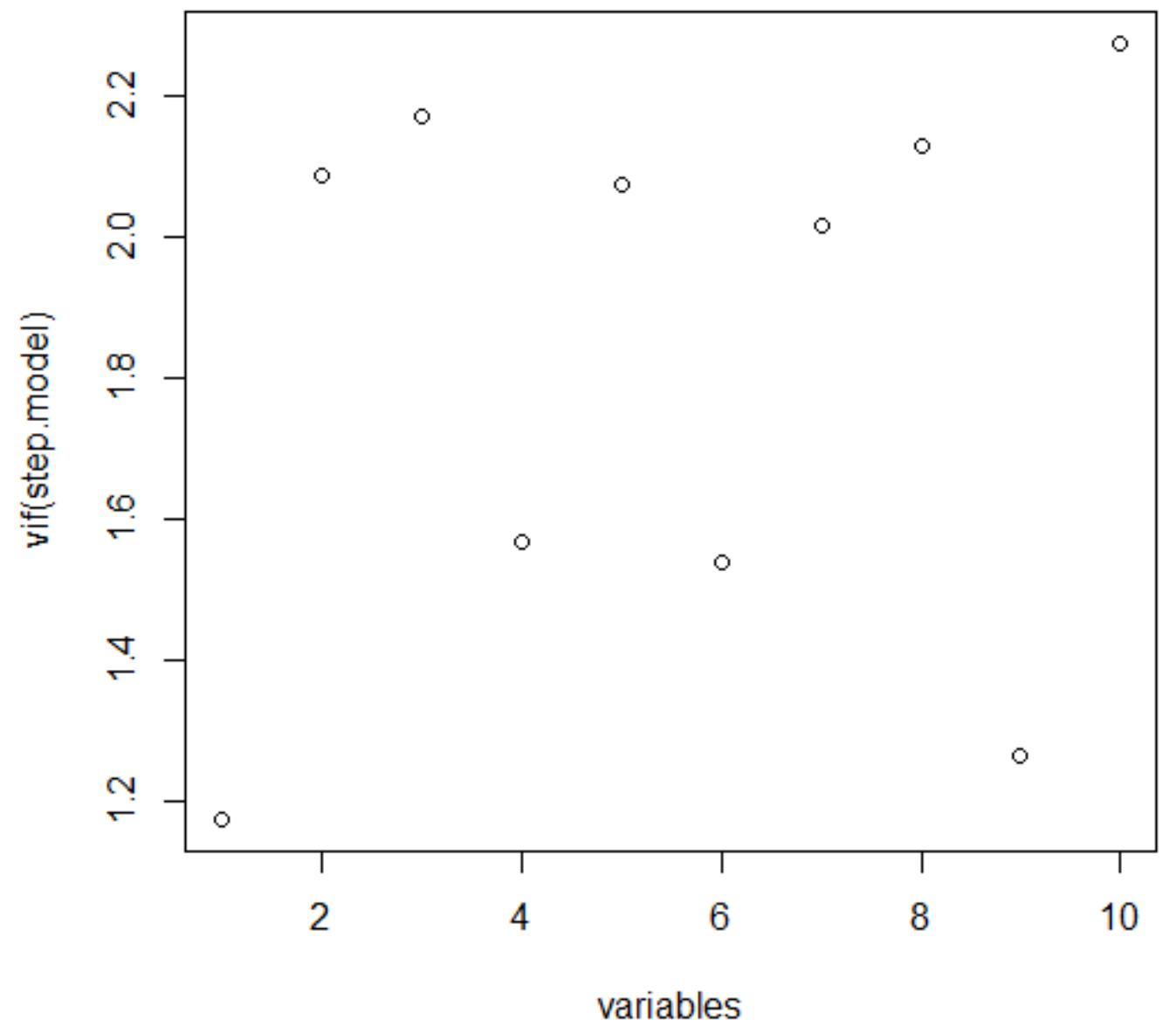


Fig.2. variance inflation factor for step-wised model

Subsequently to precisely scrutinize the prediction power of built model for unseen data we split dataset randomly to 80 percent train words and the others as test words. Then 10 -fold cross validation with partial least square analysis (PLS) results illustrated in fig.3 revealed that our best model should be used by only 4 components to predict unseen test data.

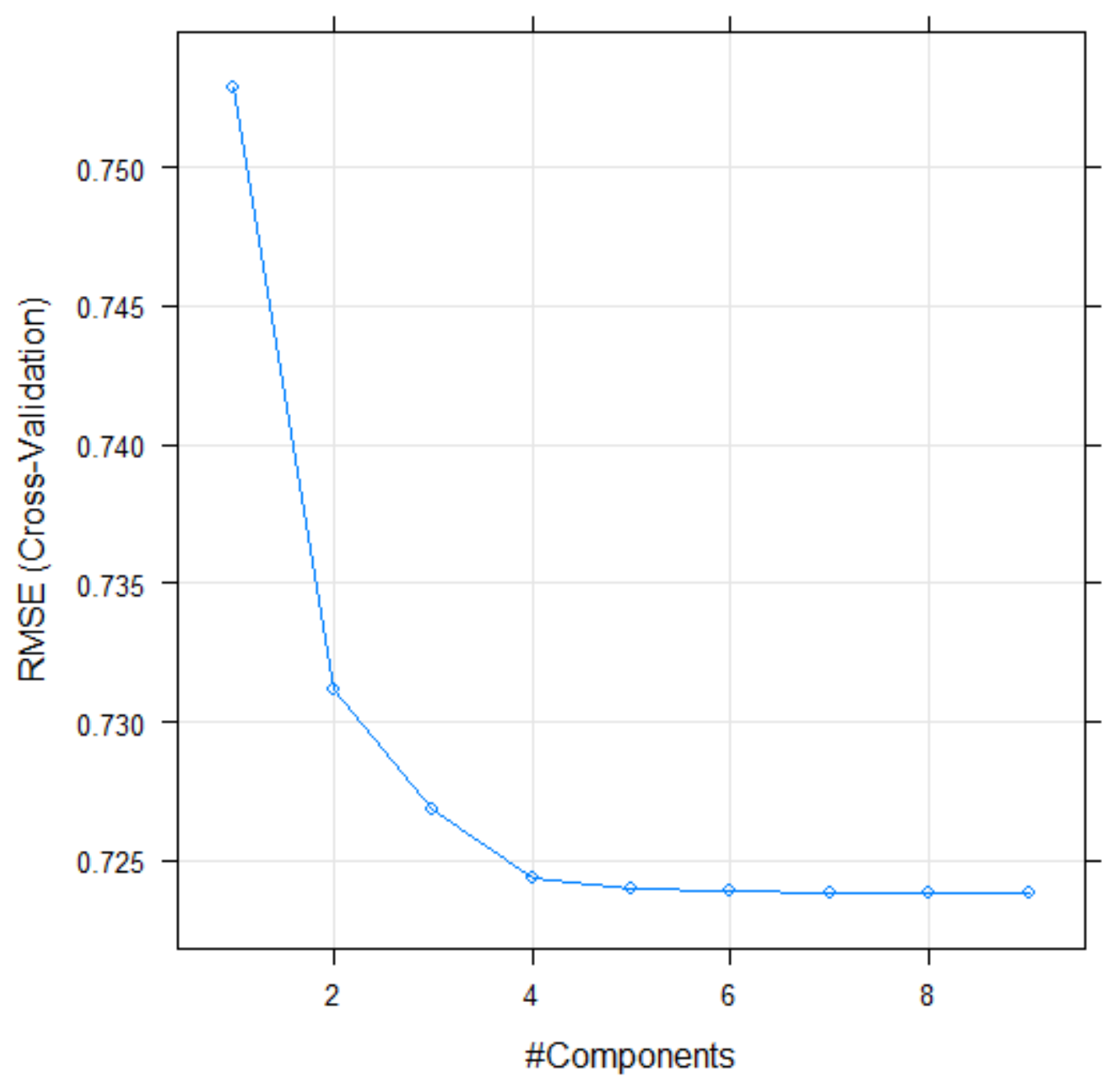


Fig.3. cross-validation with PLS results for equation 1.

Thereby, prediction the test dataset that was random selection of 20 percent of entire dataset followed. The results with PLS in Fig.4, by RMSE 0.7225 and R-square 0.5173 admirable quality of our model for prediction new test data.

Conc.M

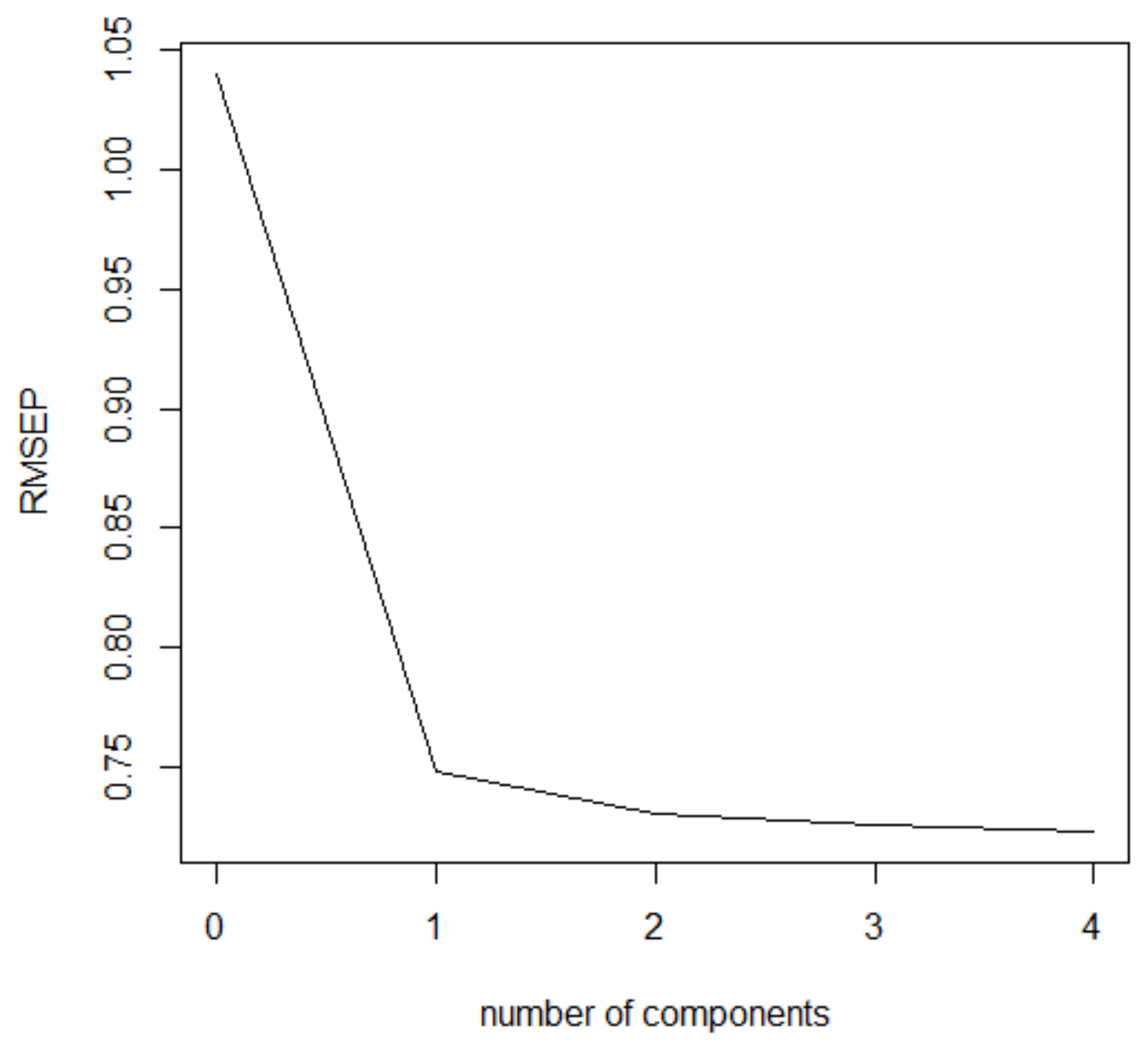

Fig.4. Prediction PLS results by 4 component

\section{Discussion}


The Lanchster dataset word norms includes multidimensional perceptual and action strengths data.In the present study after statistical selection process we found 8 variable and 6 componet were significant for prediction Brysbaer dataset concreteness values.

The three most regression coefficients in our SW-MLR model are visual mean(strength) and haptic strength and interceptive strength equal to $0.33( \pm 0.005), 0.294( \pm 0.005),-0.35( \pm 0.005)$ respectively. These parameters in our interested dataset show how much participants experiencing a word by visual perception and haptic and introspective modalities. Notwithstanding, previous studies did not show correct correspondence between object properties and tactile information. That is, tactile disadvantages for linguistic stimuli resulted by evolutionary adaption of endogenous attention(Connell and lynott,2010).

In contrast to Haptic or Visual perception, the Introspective perception is negatively correlated to concreteness which demonstrate relative importance of interceptive perception in representation of the abstract concepts.

The middle value regression coefficients demonstrate itself in Olfactory and Torso means. They are nearly equal to $0.2( \pm 0.008)$ describing a direct relationship between concreteness and these information channels. Either Olfactory or Torso strengths coefficient suggested that something that stimulate Olfactory or drive Torso part of our body is more concrete than abstract.

The lowest amount of regression coefficient values assigned to Auditory, Head and Foot-leg strengths which They have negative signs in addition to positive signs for Gustatory,Hand-arm modalities. Evidently our best model, Eq(1), after stepwise variable selection procedure, does consider head-mouth variable which means it had not enough predictive power for concreteness ratios.

\section{Conclusion}

Concreteness and abstractness of words and none words are fuzzy as well as graded property. Here we applied step- wised linear regression method to select the best model for predicting concreteness value in Brysbaer dataset by using Lancaster English frequent word norms . We found that all 11 variables of this dataset except Head-mouth parameter are useful predictors.

As a new demand to know the concreteness values of none words also infrequent words, our statistical method can pave the way for controlled experiments when choosing words as stimulus is critical. Future observational studies can prove validity of our computational model for concreteness predictions of infrequent and none words.

\section{References}


Barsalou, L. W. (1999). Perceptual symbol systems. Behavioral and Brain Sciences, 22, 577609, disc. 609-660. doi:https://doi.org/10. 1017/S0140525X99002149

Brysbaert, M., Warriner, A. B., \& Kuperman, V. (2014). Concreteness ratings for 40 thousand generally known English word lemmas. Behavior research methods, 46(3), 904-911.

Brysbaert, M., Warriner, A. B., \& Kuperman, V. (2014). Concreteness ratings for 40 thousand generally known English word lemmas. Behavior research methods, 46(3), 904-911.

Connell, L. (2019). What have labels ever done for us? The linguistic shortcut in conceptual processing. Language, Cognition and Neuroscience, 34, 1308-1318.

Connell, L., \& Lynott, D. (2010). Look but don't touch: Tactile disadvantage in processing modality-specific words. Cognition, 115(1), 1-9.

Connell, L., \& Lynott, D. (2014b). Principles of representation: Why you can't represent the same concept twice. Topics in Cognitive Science,6, 390-406.

Dohoo, I., Ducrot, C., Fourichon, C., Donald, A. and Hurnik, D. (1997), “An overview of techniques for dealing with large numbers of independent variables in epidemiologic studies", Preventive Veterinary Medicine, Vol. 29 No. 3, pp. 221-239.

Hellmann, J. H., Echterhoff, G., \& Thoben, D. F. (2013). Metaphor in embodied cognition is more than just combining two related concepts: a comment on Wilson and Golonka (2013). Frontiers in psychology, 4, 201.

Hoffman, P., \& Lambon Ralph, M. A. (2011). Reverse concreteness effects are not a typical feature of semantic dementia: evidence for the hub-and-spoke model of conceptual representation. Cerebral Cortex, 21(9), 2103-2112.

Lynott, D., Connell, L., Brysbaert, M., Brand, J., \& Carney, J. (2019). The Lancaster

Sensorimotor Norms: multidimensional measures of perceptual and action strength for 40,000 English words. Behavior Research Methods, 1-21.

Montefinese, M. (2019). Semantic representation of abstract and concrete words: A

minireview of neural evidence. Journal of neurophysiology, 121(5), 1585-1587.

Smith, L., \& Gasser, M. (2005). The development of embodied cognition: Six lessons from babies. Artificial Life, 11, 13-29.

Vigliocco, G., Meteyard, L., Andrews, M., \& Kousta, S. (2009). Toward a theory of semantic representation. Language and Cognition, 1, 219-247.

Wang J, Conder JA, Blitzer DN, Shinkareva SV. Neural representation of abstract and concrete concepts: a meta-analysis of neuroimaging studies.Hum Brain Mapp 31: 1459-1468, 2010. Wilson, M. (2002). Six views of embodied cognition. Psychonomic Bulletin \& Review, 9, 625-636.

Yao, Y., \& Zheng, X. (2020). Book Review: Embodied Cognition. Frontiers in Psychology, 11, 42. 